\section{Reações adversas a medicamentos no sistema de farmacovigilância do Brasil, 2008 a 2013: estudo descritivo}

\author{
Adverse drug reactions reported to the Brazilian \\ pharmacovigilance system from 2008 to 2013: \\ descriptive study
}

\section{Reacciones adversas a medicamentos en el sistema de farmacovigilancia de Brasil, de 2008 a 2013: estudio descriptivo}

\section{Resumo}

Analisam-se as suspeitas de reações adversas a medicamentos notificadas no sistema de farmacovigilância do Brasil (Notivisa-medicamento) entre 2008 e 2013. Trata-se de estudo descritivo cujas unidades de análises da base de dados foram as notificações e os pares medicamento-reação adversa. Foi identificado um total de 26.554 notificações, o que produziu uma taxa de notificação de 22, 8 milhões de habitantes/ano. As notificações referentes ao sexo feminino $(60,5 \%)$ prevaleceram, assim como a raça/cor branca $(58,1 \%)$. A idade variou de 0 a 112 anos (mediana = 46 anos). Quase 1/3 (32,5\%) das suspeitas de reações adversas a medicamentos ocorreram em populações vulneráveis (idosos e crianças). Foram avaliados 54.288 registros de pares de medicamento-reação adversa, em que prevaleceram as reações adversas graves (59,2\%), com destaque para as que resultaram em efeito clinicamente importante $(83,1 \%)$. Os medicamentos mais frequentes nas reações adversas a medicamentos graves pertenceram aos grupos $L$ - agentes neoplásicos e imunomodulares $(32,1 \%) e$ $J$ - anti-infeciosos gerais para uso sistêmico (27\%), ao passo que o sistema-órgão-classe mais afetado foi o relacionado a afecções da pele e distúrbios afins (23,7\%). O Notivisa-medicamento é relevante recurso para a produção de sinais de alertas e hipóteses sobre a segurança de medicamentos comercializados no Brasil. No entanto, a taxa de notificação por milhão de habitantes/ano mostrou-se muito inferior àquela descrita por países de média e alta renda.

Efeitos Colaterais e Reações Adversas Relacionados a Medicamentos; Farmacoepidemiologia; Farmacovigilância; Sistemas de Notificação de Reações Adversas a Medicamentos
Daniel Marques Mota 1

Álvaro Vigo 2

Ricardo de Souza Kuchenbecker 2

doi: $10.1590 / 0102-311 \times 00148818$

\section{Correspondência}

D. M. Mota

Agência Nacional de Vigilância Sanitária.

SIA trecho 5 - Q. especial 57, Lote 200, Bloco E, 3o andar Sala da GGREG, Brasília, DF 71205-050, Brasil.

dmarques2003@yahoo.com.br

1 Agência Nacional de Vigilância Sanitária, Brasília, Brasil. 2 Faculdade de Medicina, Universidade Federal do Rio Grande do Sul, Rio Grande do Sul, Brasil. 


\section{Introdução}

As reações adversas a medicamentos são eventos clínicos indesejáveis atribuídos aos medicamentos utilizados em doses adequadas à enfermidade e à condição clínica do paciente. Podem provocar danos graves à saúde, ocasionando, muitas vezes, admissões hospitalares e até mesmo óbito 1,2,3. Estudos norte-americanos sustentam que as reações adversas a medicamentos estão entre as dez principais causas de morte nos Estados Unidos 4 e são responsáveis por uma elevada carga financeira para os sistemas de saúde 5 .

Vários métodos de farmacovigilância têm sido utilizados para a monitoramento de reações adversas a medicamentos em condições reais 6, como forma de compensar a limitada representatividade e o poder dos ensaios clínicos 7 em evidenciar as reações adversas raras e tardias. A notificação espontânea é o método mais amplamente utilizado por diversos sistemas de farmacovigilância de base populacional 8,9. Apesar das suas reconhecidas limitações envolvendo, sobretudo, elevada subnotificação 10, esse método é particularmente importante no monitoramento de reações adversas a medicamentos raras, tardias, graves, resultantes de interações farmacológicas, decorrentes do uso contínuo de medicamento, não descritas em bula e em grupos de pacientes mais vulneráveis, como crianças e idosos 11 .

O Sistema Nacional de Notificações em Vigilância Sanitária, formulário de notificação de medicamento que ocasionou danos à saúde (Notivisa-medicamento), disponibilizado em 2008 pela Agência Nacional de Vigilância Sanitária (Anvisa), é o principal sistema de notificações espontâneas de suspeitas de reações adversas a medicamentos no Brasil. Visa, sobretudo, a agregar as notificações e, por conseguinte, gerar sinais de alertas e hipóteses sobre a segurança de medicamentos. Não identificamos estudos previamente publicados em periódicos científicos, ou mesmo em relatórios técnicos, analisando as notificações de reações adversas a medicamentos do Notivisa-medicamento. O objetivo deste estudo foi analisar as suspeitas de reações adversas a medicamentos notificadas no Notivisamedicamento no período de 2008-2013.

\section{Método}

Estudo descritivo com base de dados secundários sobre suspeitas de reações adversas a medicamentos registradas no Notivisa-medicamento e fornecida pela Anvisa, em junho de 2015. O Notivisa-medicamento compreende um sistema de vigilância passiva realizado a partir de notificações voluntárias feitas por diferentes segmentos da sociedade, sobretudo de instituições que integram a Rede Sentinela - estratégia criada pela Anvisa, em 2002, composta, principalmente, de um conjunto de hospitais públicos e privados distribuídos nas cinco regiões do país 12 .

Para fins deste estudo, utilizou-se a definição de reações adversas a medicamentos estabelecida pela Organização Mundial da Saúde (OMS), como sendo "toda resposta nociva e não intencional a um medicamento relacionada a qualquer dose normalmente utilizada no homem para profilaxia, diagnóstico e tratamento de doenças ou modificação de funções fisiológicas" ${ }^{13}$. Os critérios para definição de reações adversas a medicamentos consideradas como graves no Notivisa-medicamento são: óbito, ameaça à vida, anomalia congênita, danos que motivaram ou prolongaram a hospitalização, incapacidade persistente ou significante e efeito clinicamente importante. As reações adversas a medicamentos que resultam em outro tipo de desfecho são classificadas como não graves 14 .

As reações adversas foram agrupadas de acordo com sistema-órgão-classe (SOC) proposto pela Terminologia de Reações Adversas da OMS (WHO Adverse Reaction Terminology - WHO-ART), formado por 32 grupos 15. Para os medicamentos suspeitos, foi utilizado o sistema ATC (Anatomical Therapeutical Chemical), composto de 14 grupos de medicamentos no primeiro nível de classificação 16 . Quanto à avaliação de causalidade de cada par medicamento-reação adversa, a Anvisa adota o sistema da OMS, composto de seis categorias: definido, provável, possível, improvável, condicional/não classificado e não acessível/não classificado 14 .

A análise das reações adversas a medicamentos, no presente estudo, pressupôs a distinção de notificação e de registros no Notivisa-medicamento, sendo o termo "notificação" utilizado para se referir a um caso suspeito de reações adversas a medicamentos (pessoa afetada), ao passo que o termo "registro(s)" foi empregado para representar um par composto de medicamento-reação adversa. De 
acordo com o arranjo organizacional da base de dados da Anvisa, dois ou mais registros podem ser originados de apenas uma notificação no Notivisa-medicamento 17.

O estudo compreendeu análise de notificações/registros que atenderam aos seguintes critérios de inclusão: (a) informados no período compreendido entre 2008 e 2013, selecionados a partir da data notificada; (b) reações adversas ocorridas em território nacional e com residentes no Brasil; e (c) medicamento, como produto motivador da notificação/registro, excluídos os fitoterápicos, os homeopáticos, as vacinas e os soros hiperimunes.

Outros tipos de eventos adversos, como inefetividade terapêutica, intoxicação medicamentosa e erro de medicação foram excluídos da presente análise. Os registros com campos não preenchidos das variáveis "reações adversas" e "princípios ativos" foram igualmente excluídos.

Após a exclusão de notificações/registros que não foram objeto de interesse do estudo, a base de dados foi submetida a três etapas: (1) exploração inicial; (2) padronização geral e específica; e (3) manejo de notificações/registros repetidos. Dado o conhecimento prévio dos autores em relação ao arranjo organizacional da base de dados do Notivisa-medicamento 17 , essa base foi duplicada para ser estudada de duas maneiras distintas, isoladamente, tendo como unidades de análise as notificações dos pacientes (BDNOT) e os registros dos pares de medicamento-reação adversa (BDPAR). A exclusão de duplicidades da base de dados ocorreu na terceira etapa.

\section{Exploração inicial}

Nesta etapa, foi identificado um grande número de notificações/registros com o campo "nome do paciente" não preenchido. Essa variável é a mais importante no processo de identificação e exclusão de notificações/registros repetidos. Considerando-se esse problema, o estudo dividiu a base de dados em três bases menores, segundo as possibilidades de preenchimento das variáveis relacionadas à identificação do paciente. Esse procedimento objetivou minimizar a perda de notificações/registros a serem analisados. As três bases de dados criadas foram: (a) BD1 - notificações/registros contendo os nomes dos pacientes; (b) BD2 - notificações/registros somente com as iniciais do nome do paciente informadas, sem o nome completo; e (c) BD3 - notificações/registros com os campos nome dos pacientes e suas iniciais ausentes. Após a exclusão de notificações/registros repetidos, as três bases de dados foram unidas e divididas, de maneira a formar as bases de dados de interesse deste estudo (BDNOT e BDPAR).

\section{Padronização geral e específica}

Antes da criação das três bases de dados, a base inicial foi submetida a padronização geral e específica, que envolveu um conjunto de variáveis, denominadas de variáveis identificadoras, escolhidas para permitir a identificação de notificações/registros repetidos e únicos em cada uma das bases de dados supracitadas. Durante a padronização, mais notificações/registros foram excluídos por não atenderam aos critérios de inclusão. A padronização geral incluiu a eliminação de acentos ortográficos, cedilha e pontos, mudança das letras para maiúsculas e exclusão de excessos de espaços entre duas letras ou palavras.

O Quadro 1 apresenta as variáveis identificadoras utilizadas para cada uma das bases de dados, as regras adotadas e a padronização específica. Na persistência de notificações/registros com valores ausentes ou preenchidos como ignorados das variáveis "sexo" e "idade", após essa última padronização, esses itens foram excluídos da base de dados. Finalizada a padronização da variável "idade", decidiu-se por utilizar a variável "ano de nascimento", uma nova variável criada na base de dados, para fins da detecção de notificações/registros repetidos.

\section{Manejo de notificações/registros repetidos}

A técnica de relacionamento determinístico foi utilizada para a identificação de notificações/registros repetidos, correspondendo à combinação exata das variáveis identificadoras para cada uma das bases de dados (BD1, BD2 e BD3). A identificação e exclusão automática das notificações/registros repetidos foram realizadas em uma única etapa, por meio do uso do software SPSS, versão 18 (https:// 


\section{Quadro 1}

Variáveis identificadoras utilizadas na detecção de notificações/registros repetidos e únicos, segundo a divisão da base de dados oriunda do Notivisa-medicamento.

\begin{tabular}{|c|c|c|c|c|}
\hline Variáveis identificadoras & Regra e padronização específica & BD1 & BD2 & BD3 \\
\hline Nome do paciente & $\begin{array}{l}\text { Foram consideradas as notificações/registros com, pelo menos, dois nomes próprios. Correções } \\
\text { de erros tipográficos e de inconsistências. Termos como "Irmã" e “Senhor(a)" foram excluídos. } \\
\text { Notificações/registros iniciados com o “Recém nascido de..." ou “Lactante de..." ou "Filha(o) de..." } \\
\text { permaneceram na base de dados sem alterações. Remoção das preposições dos nomes (de, da, } \\
\text { do, das e dos). }\end{array}$ & $x$ & & \\
\hline $\begin{array}{l}\text { Iniciais do nome do } \\
\text { paciente }\end{array}$ & $\begin{array}{l}\text { Foram consideradas as notificações/registros com, pelos menos, duas letras do alfabeto. } \\
\text { Inseriram-se as iniciais que constavam no campo "nome do paciente" que não se apresentavam } \\
\text { adequadamente registradas no campo "iniciais do nome". Registros da BD1 que não atenderam às } \\
\text { regras foram inseridos na BD2. }\end{array}$ & & $x$ & \\
\hline Nome da fonte notificadora & $\begin{array}{l}\text { Exclusão de termos específicos, como "Dr" e “Dra”. As notificações/registros das bases BD1 e BD2 } \\
\text { que não atenderam às regras definidas foram inseridas na BD3. }\end{array}$ & & & $x$ \\
\hline Sexo & $\begin{array}{l}\text { Definição do sexo a partir do nome do paciente julgado ser masculino ou feminino para as } \\
\text { notificações/registros com preenchimento ignorado. Correção de algumas notificações/registros } \\
\text { quanto ao sexo. As notificações/registros com nomes duvidosos permaneceram sem alteração, } \\
\text { sendo excluídos aqueles que permanceram ignorados. }\end{array}$ & $x$ & $x$ & $x$ \\
\hline Idade & $\begin{array}{l}\text { A idade foi estabelecida na unidade "anos", variando de < } 1 \text { ano até } 112 \text { anos. Todas as idades } \\
\text { foram recalculadas a partir da seguinte fórmula no Excel: = INT(B2-A2/365,25), em que B2 é a data } \\
\text { de início do evento, A2 é a data de nascimento do paciente e 365,25 é a média de dias no ano. } \\
\text { No caso de notificações/registros com a data de início do evento não preenchida, foi utilizada a } \\
\text { data de recebimento da notificação ou a data da notificação. A idade antiga da base de dados } \\
\text { foi utilizada para complementação de notificações/Registros que permanceram com campos } \\
\text { não preenchidos no cálculo da nova idade, assim como a variável grupo de idade em relação aos } \\
\text { menores de um ano. Notificações/Registros com idade negativa foram avaliados e corrigidos. }\end{array}$ & $x$ & $x$ & $x$ \\
\hline $\begin{array}{l}\text { Cidade de ocorrência do } \\
\text { evento }\end{array}$ & $\begin{array}{l}\text { Utilizou-se cidade da instituição notificadora para preenchimento dos campos vazios. Os campos } \\
\text { com nomes de regiões administrativas do Distrito Federal foram modificados para Brasília. }\end{array}$ & $x$ & $x$ & $x$ \\
\hline Data de início do evento & $\begin{array}{l}\text { A data de recebimento pelo notificador ou a de notificação no Notivisa-medicamento } \\
\text { complementaram as notificações/Registros com campos vazios e com datas inconsistentes. }\end{array}$ & $x$ & $x$ & $x$ \\
\hline $\begin{array}{l}\text { Descrição da reações } \\
\text { adversas a medicamentos * }\end{array}$ & $\begin{array}{l}\text { Recodificação das reações adversas, com adoção do código do termo preferencial agregado do } \\
\text { WHO-ART **. Em algumas situações, um sinônimo do termo preferencial foi priorizado, como } \\
\text { agitação, em vez de agitação psicomotora. Quando havia discordância entre o código WHO-ART } \\
\text { e a descrição do evento, dava-se prioridade a esta última. Correção de erros de codificação e } \\
\text { preenchimento de campos em branco a partir da descrição do código WHO-ART. }\end{array}$ & $x$ & $x$ & $x$ \\
\hline Princípio ativo * & $\begin{array}{l}\text { Preenchimento de campos vazios por meio da variável nome comercial do medicamento. Os } \\
\text { princípios ativos foram pesquisados no bulário eletrônico da Anvisa *** e, caso não encontrados, } \\
\text { no Google. O código ATC foi pesquisado no website\#. Algumas regras foram adotadas, visando } \\
\text { à melhoria na padronização da variável. Por exemplo, para alguns fármacos, como heparina e } \\
\text { insulina, que contavam com diferentes tipos, optou-se por } \\
\text { classificá-los em um nível mais agregado. }\end{array}$ & $x$ & $x$ & $x$ \\
\hline
\end{tabular}

Anvisa: Agência Nacional de Vigilância Sanitária; ATC: Anatomical Therapeutical Chemical; BD1: notificações/registros contendo os nomes dos pacientes; BD2: notificações/registros somente com as iniciais do nome do paciente informada, sem o nome completo; e BD3: notificações/registros com os campos nome dos pacientes e suas iniciais ausentes.

* Variáveis utilizadas apenas na BDPAR.

** http://bioportal.bioontology.org/ontologies/WHO-ART/;

*** http://www.anvisa.gov.br/datavisa/fila_bula/index.asp;

\# http://www.whocc.no/atc_ddd_index/. 
www.ibm.com/), com permanência da primeira notificação/registro nas bases de dados. Os principais motivos da escolha da técnica determinística foram: (i) simplificação do processo de relacionamento de notificações/registros; (ii) praticidade e rapidez na execução; e (iii) arranjo organizacional da base de dados do Notivisa-medicamento.

Os softwares Excel 2013 (https://products.office.com/) e RecLink III, versão 3.1.6 (http://reclink. sourceforge.net/), foram utilizados na etapa de padronização das variáveis identificadoras, ao passo que o SPSS, versão 18, foi utilizado nas análises descritivas dos dados que enfocaram dois componentes: notificação (BDNOT) e pares de medicamento-reação adversa (BDPAR).

As análises incluíram a caracterização demográfica (sexo, idade, raça/cor), geográfica (regiões, estados brasileiros e municípios) e temporal (2008-2013), as fontes notificadoras mais frequentes e a identificação das principais reações adversas, medicamentos, grupos ATC, SOC afetados e pares medicamento-reação adversa. Os critérios de gravidade das reações adversas a medicamentos e as categorias de causalidade foram incluídas na caracterização dos pares de medicamento-reação adversa.

Como forma de avaliar o desempenho das regiões do país em relação às notificações de reações adversas a medicamentos no período estudado, foi calculada a taxa anual média das notificações para cada milhão de habitantes. Os dados populacionais do país e das regiões geográficas foram obtidos do Instituto Brasileiro de Geografia e Estatística (IBGE) (http://www.ibge.gov.br/home/estatistica/ populacao/projecao_da_populacao/2008/). Foi calculada a taxa de variação anual média (TVAM), que proporciona informação sobre as variações anuais acumuladas, em termos médios, entre as distintas variações de cada ano do período estudado. A TVAM é definida como:

$$
\operatorname{TVAM}(\%)=(\sqrt[n-1]{V F / V I n}-1) \times 100
$$

em que "n" representa o número de anos estudados, e VIn e VF representam o valor inicial e o valor final do período, respectivamente.

O estudo teve aprovação do comitê de ética em pesquisa do Hospital de Clínicas de Porto Alegre, da Universidade Federal do Rio Grande do Sul (parecer no 950.737/2015), e permissão institucional da Anvisa para o uso da base de dados nominais, assegurado o sigilo das informações individuais dos pacientes, notificadores e nome comercial dos medicamentos.

\section{Resultados}

Uma base de dados inicial com 58.690 notificações/registros de suspeitas de reações adversas a medicamentos foi obtida após a aplicação dos critérios de inclusão e exclusão. Foram excluídos 21.240 notificações/registros que não pertenciam ao período de tempo definido para análise e 1.996 que não se enquadraram nos demais critérios, dos quais 1.256 (62,9\%) notificações/registros foram identificados como inefetividade terapêutica e 191 (9,6\%) como intoxicação medicamentosa. Após a padronização, foram excluídos 2.826 notificações/registros com campos não preenchidos, sendo 2.511 (88,8\%) relacionados à variável "idade" e $315(11,2 \%)$ à variável "sexo".

A combinação das variáveis identificadoras utilizada para formar a BDNOT produziu 16.461 $(57,7 \%)$ notificações repetidas na BD1, ao passo que na BD2 e BD3 os percentuais corresponderam a $52,4 \%(n=13.804)$ e $49,3 \%(n=1.871)$, respectivamente. Na BDPAR, os valores para BD1, BD2 e BD3 corresponderam a, nesta ordem, 9,3\% (2.652), 5,9\% (1.565) e 4,3\% (163) registros.

Com o processo de exclusão de notificações/registros repetidos, a população do estudo, entre 2008 e 2013, foi formada por 26.554 notificações na BDNOT (BD1 = 12.069; BD2 = 12.563; e BD3 = 1.922 ) e 54.310 registros na BDPAR, correspondendo a, respectivamente, $45,2 \%$ e $92,5 \%$ do total de notificações/registros da base de dados inicial.

Outros 16 registros foram excluídos da BDPAR por problemas no preenchimento da variável "princípio ativo", resultando em uma base com 54.288 registros $(\mathrm{BD} 1=25.864 ; \mathrm{BD} 2=$ 24.794; $\mathrm{BD} 3$ = 3.630). Os motivos foram: (i) dez apresentavam vários nomes de fármacos, não se tratando de associação medicamentosa; e/ ou (ii) 12 descreviam o grupo farmacológico do medicamento. 


\section{Notificações de pacientes}

$\mathrm{Na}$ base de dados com 26.554 notificações, o sexo feminino $(60,5 \%)$ prevaleceu entre os casos com suspeitas de reações adversas a medicamentos, variando de 56\% (2008) a 62,1\% (2013). A raça/cor foi informada em 9.638 pacientes, correspondendo a 36,3\% do total das notificações. Prevaleceram a raça/cor branca e parda, respectivamente, 58,1\% e 21,2\%. A idade variou de 0 a 112 anos, com mediana igual 46 anos, com média igual 44 anos e desvio padrão de 22,7 anos. Um total de 4.430 casos (16,7\%) foi observado na faixa etária de 45 a 54 anos, e 4.304 (16,2\%) entre 55 e 64 anos de idade. Quase 1/3 $(32,5 \%)$ das suspeitas de reações adversas a medicamentos ocorreram em populações mais vulneráveis, particularmente os idosos ( $\geq 65$ anos), que representaram $19,6 \%$, e as crianças ( $\leq 14$ anos), com um percentual menor de $12,9 \%$.

A origem geográfica das notificações foi principalmente das regiões Sudeste (59,7\%) e Sul (17\%). Essas regiões apresentaram as taxas mais elevadas de notificação por um milhão de habitantes (Tabela 1). Os cincos estados com maior percentual de notificações foram, nesta ordem: São Paulo ( $\mathrm{n}=$ 10.331; 38,9\%), Minas Gerais ( $\mathrm{n}=2.737$; 10,3\%), Rio de Janeiro ( $\mathrm{n}=2.663$; $10 \%$ ), Rio Grande do Sul ( $\mathrm{n}=2.570 ; 9,7 \%)$ e Ceará $(\mathrm{n}=1.781 ; 6,7 \%)$. Não houve notificações de casos provenientes do Amapá. Entre 2008 e 2013, observou-se que 418 municípios estão representados na população com suspeitas de reações adversas a medicamentos, correspondendo a 7,5\% do total de 5.565 municípios brasileiros (ano de 2010). São Paulo ( $n=7.476 ; 28,2 \%$ ), Rio de Janeiro ( $n=2.594 ; 9,8 \%$ ) e Belo Horizonte ( $\mathrm{n}=2.039 ; 7,7 \%)$ foram os municípios mais prevalentes quanto ao local de ocorrência das reações adversas a medicamentos.

A fonte notificadora foi informada em $73,6 \%(\mathrm{n}=19.548)$ dos casos registrados na BDNOT. Desse total, 55,1\% provieram da Rede Sentinela, 15,5\% de hospitais e 1,2\% das vigilâncias sanitárias (Anvisa, vigilâncias estaduais, municipais e distrital). Os laboratórios farmacêuticos contribuíram com 0,7\% das notificações registradas no Notivisa-medicamento. Ao longo do período estudado, houve um aumento crescente de notificações e registros no Notivisa-medicamento, resultando em TVAM de 23,2\% e 20,4\%, respectivamente. Obteve-se uma mediana de 2,1 registros para cada notificação, variando de 1,8 (2013) a 2,2 (2009 e 2011) (Tabela 2).

\section{Registros de pares medicamento-reação adversa}

No período de 2008 a 2013, foram identificados 839 princípios ativos (108 associações medicamentosas) responsáveis por 765 suspeitas de reações adversas, resultando na formação de 15.013 pares de medicamento-reação adversa diferentes na BDPAR. A distribuição anual dessas informações está demonstrada nas Tabelas 2 e 3. Em 2013, houve um aumento de 2,5 vezes no número de registros quando comparado ao ano de 2008. Ao mesmo tempo, houve uma redução na razão de reações adversas a medicamentos graves: não graves em -16,8\% (Tabela 2).

Tabela 1

Taxa anual média (TAM) de notificações registradas no Notivisa-medicamento por regiões do Brasil, 2008-2013.

\begin{tabular}{lccc}
\hline Região & Total de notificações & $\begin{array}{c}\text { Número médio anual de } \\
\text { notificações }\end{array}$ & $\begin{array}{c}\text { Número médio anual de } \\
\text { habitantes }\end{array}$ \\
\hline Norte & 1.635 & 273 & 15.649 .261 \\
Nordeste & 4.311 & 719 & 54.262 .449 \\
Sudeste & 15.850 & 2.642 & 81.886 .921 \\
Centro-oeste & 235 & 39 & 14.161 .671 \\
Sul & 4.523 & 754 & 28.014 .423 \\
Brasil & 26.554 & 4.427 & 13,2 \\
\hline
\end{tabular}


Tabela 2

Distribuição anual de registros, notificações e número de princípios ativos e reações adversas diferentes no Notivisa-medicamento, 2008 -2013.

\begin{tabular}{|c|c|c|c|c|c|c|}
\hline \multirow[t]{2}{*}{ Ano } & \multirow[t]{2}{*}{ Total de registros } & \multirow[t]{2}{*}{ Total de notificações } & \multirow{2}{*}{$\begin{array}{c}\text { Número de princípios } \\
\text { ativos * }\end{array}$} & \multirow{2}{*}{$\begin{array}{c}\text { Número de reações } \\
\text { adversas }\end{array}$} & \multicolumn{2}{|c|}{ Gravidade dos registros } \\
\hline & & & & & Grave (\%) & Não grave (\%) \\
\hline 2008 & 4.864 & 2.322 & 380 & 356 & $2.954(60,7)$ & $1.910(39,3)$ \\
\hline 2009 & 6.560 & 2.988 & 418 & 326 & $3.588(54,7)$ & $2.972(45,3)$ \\
\hline 2010 & 8.582 & 4.093 & 479 & 411 & $4.879(56,9)$ & $3.703(43,1)$ \\
\hline 2011 & 11.220 & 5.164 & 513 & 433 & $6.731(60,0)$ & $4.489(40,0)$ \\
\hline 2012 & 10.834 & 5.387 & 476 & 418 & $7.088(65,4)$ & $3.746(34,6)$ \\
\hline 2013 & 12.228 & 6.600 & 511 & 475 & $6.902(56,4)$ & $5.326(43,6)$ \\
\hline Total & 54.288 & 26.554 & - & - & $32.142(59,2)$ & $22.146(40,8)$ \\
\hline
\end{tabular}

* Cada associação medicamentosa foi contada como um princípio ativo.

Quanto à gravidade, prevaleceram as reações adversas graves $(59,2 \% ; \mathrm{n}=32.142)$ (Tabela 2$)$, com destaque para as que resultaram em efeito clinicamente importante $(83,1 \%)$, motivaram ou prolongaram a hospitalização $(9,2 \%)$ e ameaça à vida (4,7\%). Casos de anomalias congênitas e óbitos atribuídos ao uso de medicamentos foram constatados em $22(0,1 \%)$ e $585(1,8 \%)$ registros de par medicamento -reação adversa, respectivamente. A Tabela 3 apresenta a quantidade de pares de medicamento-reação adversa diferentes e os cinco pares mais prevalentes, segundo gravidade e ano de notificação. Há certa homogeneidade na predominância dos principais pares ao longo dos anos, principalmente para aqueles enquadrados como reações adversas a medicamentos graves.

Foi constatada uma distribuição equânime entre os grupos da classificação ATC dos medicamentos para as reações adversas a medicamentos graves e não graves, com a presença dos 14 grupos da ATC. Os mais frequentes nas reações adversas a medicamentos graves foram: $\mathrm{L}$ - agentes neoplásicos e imunomodulares (32,1\%), J - anti-infeciosos gerais para uso sistêmico (27\%) e N - sistema nervoso (12,9\%). Naquelas não graves, os grupos ATC corresponderam a, nesta ordem: J - anti-infeciosos gerais para uso sistêmico (26,8\%), L - agentes neoplásicos e imunomodulares (19,5\%) e N - sistema nervoso $(18,6 \%)$.

Os medicamentos docetaxel $(9,1 \%)$, vancomicina $(4,1 \%)$, paclitaxel $(3,6 \%)$, ciprofloxacino $(2,6 \%)$, dipirona $(2,4 \%)$, carboplatina $(1,9 \%)$ e oxiplatina $(1,9 \%)$ foram os sete fármacos mais frequentemente suspeitos de envolvimento com as reações adversas a medicamentos graves. Já os medicamentos dipirona (3,8\%), paclitaxel $(3,6 \%)$, vancomicina (3,4\%), morfina (3\%), ciprofloxacino (3\%), tramadol (3\%) e docetaxel (2,9\%) foram os sete fármacos suspeitos mais frequentes nas reações adversas a medicamentos não graves.

As 11 reações adversas mais frequentemente descritas, segundo a gravidade, estão apresentadas na Tabela 4. Os três SOC mais afetados nas reações adversas a medicamentos graves foram: afecções da pele e distúrbios afins (23,7\%), distúrbios do estado geral (13,5\%) e distúrbios do sistema gastrointestinal (11,9\%). Os principais SOC encontrados para as reações adversas a medicamentos não graves não foram diferentes daqueles que caracterizaram as reações adversas a medicamentos graves, prevalecendo afecções da pele e distúrbios afins (36,1\%), distúrbios do estado geral (14,4\%) e distúrbios do sistema gastrointestinal $(13,9 \%)$.

Em 82,6\% dos registros de pares medicamento-reações adversa, a reação adversa notificada estava descrita na bula do medicamento, com um total de 35.194 (64,8\%) registros com essa informação. Casos simultâneos de reações adversas a medicamentos graves e não descritas na bula ocorreram em 3.829 (7\%) casos do total de registros. O uso de medicamentos concomitantes estava presente em 70\% de 38.630 dos registros que continham esse dado. A informação sobre interação medicamentosa foi identificada em 32.440 registros, dos quais em 13,3\% $(n=4.318)$ foi considerada a possibilidade de ocorrência desse fenômeno. Quatorze vias de administração dos medicamentos foram mencionadas em 43.526 (80,2\%) registros, com predominância da intravenosa $(75,3 \%)$ e oral $(20,6 \%)$. 


\section{Tabela 3}

Quantidade anual dos pares medicamento-reação adversa e os cinco pares mais prevalentes no Notivisa-medicamento, $2008-2013$.

\begin{tabular}{|c|c|c|c|}
\hline \multirow[t]{2}{*}{ Ano } & \multirow[t]{2}{*}{ Total de pares } & \multicolumn{2}{|l|}{ Principais pares * } \\
\hline & & Grave & Não grave \\
\hline \multirow[t]{5}{*}{2008} & 2.824 & Docetaxel-Hiperemia da pele $(n=53 ; 1,8 \%)$ & Dipirona-Eritema $(n=27 ; 1,4 \%)$ \\
\hline & & Docetaxel-Dispnéia $(n=46 ; 1,6 \%)$ & Vancomicina-Prurido $(n=20 ; 1,0 \%)$ \\
\hline & & Docetaxel-Dor no peito $(n=32 ; 1,1 \%)$ & Dipirona-Prurido ( $n=20 ; 1,0 \%)$ \\
\hline & & Docetaxel-Dorsalgia $(n=29 ; 1,0 \%)$ & Vancomicina-Eritema $(n=18 ; 0,9 \%)$ \\
\hline & & Paclitaxel-Dispneia $(n=19 ; 0,6 \%)$ & Ciprofloxacina-Prurido $(n=16 ; 0,8 \%)$ \\
\hline \multirow[t]{5}{*}{2009} & 3.261 & Docetaxel-Hiperemia da pele $(n=103 ; 2,9 \%)$ & Morfina-Prurido $(n=44 ; 1,5 \%)$ \\
\hline & & Docetaxel-Dispneia $(n=102 ; 2,8 \%)$ & Dipirona-Prurido $(n=40 ; 1,3 \%)$ \\
\hline & & Docetaxel-Dor no peito $(n=85 ; 2,4 \%)$ & Tramadol-Náuseas ( $n=23 ; 0,8 \%)$ \\
\hline & & Docetaxel-Dorsalgia $(n=42 ; 1.2 \%)$ & Cefalotina-Prurido $(n=21 ; 0,7 \%)$ \\
\hline & & Vancomicina-Prurido $(\mathrm{n}=26 ; 0,7 \%) * *$ & Oseltamivir-Vômitos ( $\mathrm{n}=21 ; 0,7 \%$ ) \\
\hline \multirow[t]{5}{*}{2010} & 4.197 & Ácido diatrizóico+Diatrizoato de meglumina-Urticária ( $n=37 ; 0,8 \%)$ & Morfina-Pele seca $(n=64 ; 1,7 \%)$ \\
\hline & & Vancomicina-Prurido $(n=37 ; 0,8 \%)$ & Morfina-Prurido $(n=43 ; 1,2 \%)$ \\
\hline & & Ácido diatrizóico+Diatrizoato de meglumina-Prurido $(n=34 ; 0,7 \%)$ & Docetaxel-Hiperemia da pele $(n=35 ; 0,9 \%)$ \\
\hline & & Vanconmicina-Erupção cutânea $(n=31 ; 0,6 \%)$ & Docetaxel-Dorsalgia $(n=28 ; 0,8 \%)$ \\
\hline & & Docetaxel-Hiperemia da pele $(n=30 ; 0,6 \%)$ & Dipirona-Prurido ( $n=28 ; 0,8 \%)$ \\
\hline \multirow[t]{5}{*}{2011} & 5.073 & Docetaxel-Hiperemia da pele $(n=144 ; 2,1 \%)$ & $\begin{array}{l}\text { Ácido diatrizóico+Diatrizoato de meglumina- } \\
\text { Urticária }(\mathrm{n}=95 ; 2,1 \%)\end{array}$ \\
\hline & & Docetaxel-Dor no peito $(n=120 ; 1,8 \%)$ & Furosemida-Hipotassemia ( $n=49 ; 1,1 \%)$ \\
\hline & & Docetaxel-Dispneia $(n=119 ; 1,8 \%)$ & Prurido-Morfina $(n=45 ; 1,0 \%)$ \\
\hline & & Vancomicina-Prurido $(n=52 ; 0,8 \%)$ & Dorsalgia-Docetaxel $(n=42 ; 0,9 \%)$ \\
\hline & & Docetaxel-Dor renal $(n=47 ; 0,7 \%)$ & Hiperemia da pele-Docetaxel $(n=27 ; 0,6 \%) \star \star \star$ \\
\hline \multirow[t]{5}{*}{2012} & 4.614 & Docetaxel-Hiperemia da pele $(n=142 ; 2,0 \%)$ & Morfina-Prurido $(n=59 ; 1,6 \%)$ \\
\hline & & Paclitaxel-Choque anafilático $(n=110 ; 1,6 \%)$ & Paclitaxel-Choque anafilático ( $n=56 ; 1,5 \%)$ \\
\hline & & Docetaxel-Dispneia ( $\mathrm{n}=89 ; 1.3 \%)$ & Dipirona-Prurido ( $n=38 ; 1,0 \%)$ \\
\hline & & Docetaxel-Dor no peito- $(\mathrm{n}=85 ; 1,2 \%)$ & Iopramida-Prurido ( $\mathrm{n}=33 ; 0,9 \%$ ) \\
\hline & & Vancomicina-Prurido $(n=69 ; 1,0 \%)$ & Vancomicina-Prurido $(n=33 ; 0,9 \%)$ \\
\hline \multirow[t]{5}{*}{2013} & 5.085 & Docetaxel-Hiperemia da pele $(n=129 ; 1,9 \%)$ & Paclitaxel-Choque anafilático $(n=188 ; 3,5 \%)$ \\
\hline & & Docetaxel-Dispneia $(n=118 ; 1,7 \%)$ & Docetaxel-Choque anafilático $(n=107 ; 2,0 \%)$ \\
\hline & & Docetaxel-Dor renal $(n=100 ; 1,4 \%)$ & Morfina-Prurido $(n=94 ; 1,8 \%)$ \\
\hline & & Docetaxel-Dor no peito $(n=85 ; 1,2 \%)$ & Oxaliplatina-Choque anafilático $(n=53 ; 1,0 \%)$ \\
\hline & & Morfina-Prurido $(n=71 ; 1,0 \%)$ & Vancomicina-Prurido $(\mathrm{n}=52 ; 1,0 \%)$ \\
\hline
\end{tabular}

* Frequência absoluta e relativa dos pares medicamento-reação adversa, segundo gravidade e ano de notificação no Notivisa-medicamento. Os valores totais dos pares de reações adversas graves e não graves para cada ano estão na Tabela 2;

** Par Docetaxel-Dor abdominal $(n=26 ; 0,7 \%)$ apresentou os mesmos valores;

*** Par Dipirona-Prurido $(n=27 ; 0,6 \%)$ apresentou os mesmos valores.

A variável que representa o "nexo causal" estava preenchida em 30.060 registros (55,4\%). Em 29.377 (97,7\%) desses registros, a avaliação de causalidade do par medicamento-reação adversa foi estabelecida. Predominaram as seguintes categorias de causalidade: possível (46,7\%), provável (44,1\%) e não acessível/não classificavél (3,9\%). As categorias "definida” e "improvável” representaram 1,4\% e 2,6\% dos pares, respectivamente. Um total de 683 (2,3\%) registros apresentou o conteúdo preenchido com outras três opções: investigado $(n=346)$, indefinido $(n=175)$ e não relacionado $(n=162)$.

Observou-se uma redução acentuada na quantidade de registros com nexo causal definido, provável e possível em relação ao total de registros no Notivisa-medicamento, da ordem de $83 \%$ (4.032/4.864) em 2008 para 21,1\% (2.587/12.228) em 2013. O menor valor foi registrado em 2012, com $18,1 \%$ (1.959/10.834), com mediana no período de $67,1 \%$. 
Reações adversas mais frequentes registradas no Notivisa-medicamento, segundo a gravidade, 2008-2013 ( $\mathrm{N}=54.288)$.

\begin{tabular}{|c|c|c|c|c|c|}
\hline \multicolumn{3}{|c|}{ Graves $(n=32.142)$} & \multicolumn{3}{|c|}{ Não graves $(n=22.146)$} \\
\hline Reação adversa & $\mathbf{n}$ & $\%$ & Reação adversa & $\mathbf{n}$ & $\%$ \\
\hline Prurido & 2.595 & 8,1 & Prurido & 3.395 & 15,3 \\
\hline Dispneia & 1.511 & 4,7 & Erupção cutânea & 1.385 & 6,3 \\
\hline Erupção cutânea & 1.459 & 4,5 & Hiperemia da pele & 1.309 & 5,9 \\
\hline Hiperemia da pele & 1.405 & 4,4 & Eritema & 1.149 & 5,2 \\
\hline Eritema & 1.077 & 3,4 & Náuseas & 1.045 & 4,7 \\
\hline Vômitos & 973 & 3,0 & Urticária & 1.029 & 4,6 \\
\hline Vasodilatação & 886 & 2,8 & Vasodilatação & 956 & 4,3 \\
\hline Náuseas & 862 & 2,7 & Vômitos & 914 & 4,1 \\
\hline Urticária & 852 & 2,6 & Choque anafilático & 673 & 3,0 \\
\hline Dor no peito & 654 & 2,0 & Flebite & 441 & 2,0 \\
\hline Diarreia & 582 & 1,8 & Diarreia & 439 & 2,0 \\
\hline
\end{tabular}

\section{Discussão}

Em que pese o aumento das notificações no período estudado (2008-2013), nosso estudo apontou uma taxa de notificação muito baixa para o país (22,8 notificações/milhão de habitantes), se comparada àquelas observadas em países como Nova Zelândia, Suécia, Austrália e Suíça, que apresentaram mais de trezentas notificações por milhão de habitantes no período de 2000 a 2009 18. O sistema de farmacovigilância da África do Sul registrou uma taxa de 77 notificações por milhão de habitantes 19 em 2011, ao passo que, em Portugal, a taxa média anual foi de 110 notificações por milhão de habitantes no período de 2009-2011 11.

A subnotificação é mais evidente se estratificada entre as cinco regiões brasileiras, variando, nas regiões Centro-oeste e Nordeste, de, respectivamente, 2,7 e 13,2 notificações/milhão de habitantes a 27,0 e 32,3 notificações/milhão de habitantes nas regiões Sul e Sudeste. Resultado de diferentes fatores socioeconômicos e sanitários, essa heterogeneidade provavelmente também provém da distribuição e participação dos hospitais da Rede Sentinela, demonstrando não apenas a sua relevância, mas também a sua preponderância nas notificações de reações adversas a medicamentos no país.

Os estados de São Paulo $(\mathrm{n}=54)$, Minas Gerais $(\mathrm{n}=21)$, Rio de Janeiro $(\mathrm{n}=18)$ e Santa Catarina $(\mathrm{n}=18)$ possuem a maior frequência de instituições na Rede Sentinela, de um total de 193 (lista atualizada em abril de 2013) 20. O Estado do Rio Grande do Sul está em quarto lugar, com 12 hospitais. O Ceará $(n=8)$, na sexta colocação, conta também com um sistema de farmacovigilância local bastante proativo 21. À época, não havia hospitais dos estados de Amapá e Roraima na Rede Sentinela. Esses fatos apontam para uma importante influência do perfil de notificações/registros originários dos Hospitais Sentinela na caracterização do perfil de casos do Notivisa-medicamento em relação aos fármacos mais frequentes, frequência dos pares de medicamentos-reações adversas, prevalência das reações e respectiva gravidade, podendo ser considerado como possível viés dos achados do estudo.

As notificações de reações adversas a medicamentos advindas dos hospitais são relevantes porque, com frequência, os novos medicamentos comercializados, em geral, são primeiramente utilizados nessa modalidade assistencial, o que, somado à condição aguda experimentada pelo paciente, aumenta a probabilidade de ocorrência de reações adversas, permitindo também que essas reações sejam detectadas logo no seu início. Consequentemente, as notificações espontâneas tendem a ser mais acuradas e completas 21,22 , visto que parte importante das admissões hospitalares estão associadas com reações adversas a medicamentos 23 .

É digna de nota a pequena participação das notificações provenientes dos laboratórios farmacêuticos. Conforme resolução da Anvisa, desde 2009, essa fonte notificadora é obrigada a notificar as reações adversas a medicamentos graves 24 . As reações adversas a medicamentos classificadas como 
graves representaram 62\% dos 193 registros identificados como de origem nos laboratórios farmacêuticos. Diferentemente dos nossos achados, um estudo português encontrou que as notificações dos laboratórios farmacêuticos representaram quase 47\% de todos os registros analisados entre 2009 e 2011 11. É possível que as notificações oriundas dos laboratórios que se concentram no estado de São Paulo tenham sido feitas ao sistema de vigilância de reações adversas a medicamentos da secretaria de saúde daquele estado e, portanto, não estejam contempladas no Notivisa-medicamento. Não são conhecidos estudos e relatórios sobre as notificações de reações adversas a medicamentos oriundas de laboratórios farmacêuticos no Brasil.

O objetivo primordial de sistemas de farmacovigilância envolve, para além da geração de informações sobre a segurança de medicamentos, a avaliação das relações de causa e efeito entre o fármaco em questão e as reações adversas a medicamentos. No Notivisa-medicamento, cada par medicamento-reação adversa é revisado individualmente por técnicos da Anvisa, para que a relação causal possa ser estabelecida. Causa espécie a relação de causa-efeito não ter sido realizada em quase metade das notificações (44,6\%), envolvendo, principalmente, os anos 2012 e 2013. Esse achado provavelmente explica a acentuada redução na quantidade de pares medicamento-reação adversa classificados, do ponto de vista da relação causal, como "definidos", "prováveis" e "possíveis", verificados principalmente nos anos 2012 e 2013. Esses achados levam a inferir um comprometimento significativo na geração de sinais de segurança e, por conseguinte, sérias limitações no planejamento e adoção de medidas regulatórias oportunas e adequadas para um efetivo monitoramento e controle de reações adversas a medicamentos.

A vigilância e o monitoramento de pessoas que fazem uso de medicamentos englobam, necessariamente, a produção de dados e análises das informações sobre reações adversas a medicamentos dos sistemas de farmacovigilância. Por exemplo, o sistema de notificações de eventos adversos da Food and Drug Administration (AERS/FDA) dos Estados Unidos tem proporcionado evidências de que certos medicamentos aprovados para uso produziram graves problemas de segurança. Alguns deles foram retirados do mercado com base na totalidade ou em parte das notificações de eventos adversos 25 .

Considerando o predomínio das reações adversas graves $(59,2 \% ; \mathrm{n}=32.142)$ (Tabela 2), destacamse as que resultaram em efeito clinicamente importante $(83,1 \%)$, motivaram ou prolongaram a hospitalização $(9,2 \%)$ e ameaça à vida (4,7\%). Esses achados diferem, em certa medida, de estudo espanhol que apontou maior frequência de hospitalizações (48\%) e ameaça à vida (24\%) e valores bem menores para efeito clinicamente importante (20\%) no período de dez anos (1998-2008) 26.

Constatou-se grande diversidade de medicamentos supostamente relacionados às reações adversas, pois todos os catorze grupos de classificação ATC foram representados no estudo. Os dados mostraram que os três grupos de medicamentos predominantes foram os mesmos em ambas as reações graves e não graves ( $\mathrm{L}$ - agentes neoplásicos e imunomodulares, $\mathrm{J}$ - anti-infeciosos gerais para uso sistêmico e N - sistema nervoso). No conjunto, esses grupos representaram respectivamente $72 \%$ e 64,9\% do total de medicamentos relacionados com reações adversas. Resultados semelhantes foram constatados em estudo com dados do sistema de notificações espontâneas de Portugal 11 .

A facilidade no reconhecimento de situações clínicas que comprometem o sistema tegumentar (pele e anexos) do paciente também é constatada nos casos de suspeitas de reações adversas a medicamentos. Necrólise epidérmica tóxica e síndrome de Stevens Johnson são exemplos clássicos de reações adversas a medicamentos graves que, ao comprometerem o sistema tegumentar de maneira específica, facilitam o diagnóstico 27 . Nessa direção, muitas reações adversas a medicamentos não graves frequentemente notificadas (como prurido, erupção cutânea e hiperemia da pele) constituem outra situação que ajuda a explicar as afecções da pele e distúrbios afins como o principal SOC afetado encontrado em nosso estudo e também descrito nos sistemas de farmacovigilância de outros países 28 .

Especial atenção deve ser dada na decisão sobre se um relato de caso preenche os critérios definidos para reações adversas a medicamentos grave. Nesses casos, deve-se agilizar sua elegibilidade para notificação às autoridades reguladoras. Dificuldades podem ocorrer no reconhecimento da gravidade, principalmente na ausência de critérios claros, como o que motivou ou prolongou a hospitalização. Para reduzir essa incerteza, o Council for International Organizations of Medical Sciences (CIOMS) propôs uma lista com 836 (7\%) termos, de um total de 12 mil, que devem sempre ser considerados "graves", como broncoespasmo, ulceração mucocutânea, flebite e síndrome de Stevens Johnson 29. Vários registros analisados do Notivisa-medicamento não atenderam à lista, pois termos 
que deveriam ter sido classificados como graves, a exemplo de choque anafilático, flebite, angioedema, agranulocitose, entre outros, foram descritos como não graves.

A identificação de fatores de predisposição para reações adversas a medicamentos pelos sistemas de farmacovigilância é essencial para a segurança dos pacientes. Os grupos de idade mais vulnerável (como crianças e idosos), doenças hepáticas e renais, polifarmácia e sexo feminino são alguns dos fatores que sugerem maior susceptibilidade às reações adversas a medicamentos 30 . Nos dados do Notivisa-medicamento, predominaram a população feminina e o uso de medicamentos concomitantes. Estudos que analisaram dados de sistemas de notificações espontâneas também encontraram resultados semelhantes quanto ao predomínio de alguns dos fatores de predisposição 11,21,25.

Dados sobre grupos étnicos também devem ser monitorados pelos sistemas de farmacovigilância. Os pacientes de vários desses grupos têm riscos diferenciados para reações adversas a medicamentos, o que pode ser um determinante de danos no tratamento do paciente, seja por uma medida substituta da constituição genética, seja por fatores culturais que alteram o risco da ocorrência do problema 31 . A título exemplificativo, indivíduos negros em uso de fármacos cardiovasculares são mais susceptíveis de desenvolvimento de reações adversas a medicamentos do que não negros, segundo a conclusão dos autores do estudo 31. Em nosso estudo, apesar da escassa informação, a população negra e parda representou 1/3 dos casos notificados no Notivisa-medicamento, o que pode ser ainda maior, dada a composição étnica variável de cada região do país. No sistema americano, sequer há um campo disponível no formulário para a coleta dessa informação 25 .

A utilização de dados provenientes de sistemas de farmacovigilância baseados em notificações espontâneas apresenta várias limitações conhecidas que dificultam o estabelecimento de inferências de causalidade, como a ocorrência de elevada subnotificação, problemas na qualidade dos dados, vieses de notificação, dificuldades no reconhecimento de reações adversas a medicamentos e desconhecimento do total de pessoas expostas aos medicamentos, mas que apresentam a mesma condição clínica em análise 10,25,32. Nosso estudo apurou elevada subnotificação se comparada com parâmetros internacionais de sistemas de farmacovigilância (300/1 milhão de habitantes/ano) 33. Foram também identificadas incompletudes de campos importantes do formulário do Notivisa-medicamento, como sexo, idade e raça/cor, dificultando uma caracterização mais acurada da realidade do país. Tornam-se necessários, pois, a criação e o monitoramento de mecanismos que minimizem esses problemas, pois as reações adversas a medicamentos figuram entre as principais causas de morbidade e mortalidade em diversos países $1,2,3$.

As limitações dos sistemas de notificações espontâneas não devem resultar no desuso dos dados, pois, muitas vezes, o início da vigilância ativa de reações adversas a medicamentos tem como ponto de partida as evidências identificadas pelo acúmulo de notificações espontâneas 25 . A relação entre o uso de clopidrogel com a ocorrência de púrpura trombocitopênica trombótica, por exemplo, se deu a partir de relatos espontâneos que relacionaram a ticlopidina, um fármaco da mesma classe do clopidogrel, com tal reação adversa 25,34. Ademais, os sistemas de notificações espontâneas são provavelmente o método de melhor relação custo-efetividade para o monitoramento de reações adversas a medicamentos graves e raras não descobertas por ocasião da realização dos ensaios clínicos 32 .

Chama atenção que a base de dados do Notivisa-medicamento apresenta uma peculiaridade na estrutura de notificação de casos suspeitos de reações adversas a medicamentos em comparação com aquelas oriundas de sistemas de vigilância epidemiológica, pois não obedece ao seguinte arranjo tradicional: uma notificação $=$ um registro $=$ um paciente. Uma notificação advinda de um único paciente pode resultar em mais de um registro na base de dados, pois a sua organização no Notivisa-medicamento baseia-se em pares de fenômenos, como medicamento-reação adversa ou reação adversa-classificação da sua gravidade. Cada arranjo consta como um registro na base de dados. Assim, os registros oriundos de um único paciente se diferenciam apenas nas informações peculiares para cada par de fenômeno. As informações, como idade e sexo, entre outras, se repetem em cada um dos registros 17. Esse tipo de arranjo contribui para que a quantidade de notificações repetidas na base do Notivisa-medicamento seja elevada. Isso possivelmente não se observa em outros sistemas de vigilância de saúde pública que se apresentam como arranjo tradicional.

A preparação da base de dados e a padronização das variáveis identificadoras constituíram as atividades críticas e que demandaram mais trabalho no presente estudo, devido à inconsistência $\mathrm{e}$ à carência de dados de parte das informações da base de dados do Notivisa-medicamento. Erros de 
preenchimento, dados incompletos e campos não preenchidos dificultam o relacionamento de notificações/registros na etapa de detecção de duplicidades e, por conseguinte, podem artificialmente produzir sinais de segurança não verdadeiros.

Buscou-se identificar e corrigir os erros de preenchimento e os dados incompletos por meio de inspeção visual e comparação de variáveis. No caso dos campos incompletos, optou-se pela exclusão de notificações/registros com variáveis identificadoras não preenchidas, evitando falha no pareamento de duas ou mais notificações/registros que correspondessem a uma mesma pessoa (falsos-negativos). Não houve diferença significativa no uso da variável "ano de nascimento" em relação à variável "idade”, no que tange ao número de pares repetidos e únicos formados. Os resultados apresentados neste estudo podem não estar totalmente livres de erros, principalmente no que tange às variáveis "nome do paciente", "iniciais do nome" e "nome do notificador", que foram utilizadas como fundamentais no processo de identificação de notificações/registros repetidos.

O Notivisa-medicamento é importante recurso para produção de informações sobre farmacovigilância no Brasil, devendo ser utilizado para identificar padrões desconhecidos sobre perfil de segurança dos medicamentos. Nosso estudo realizou análise inédita de notificações/registros do Notivisa-medicamento que, embora seja o principal repositório de dados sobre reações adversas a medicamentos no Brasil, representa apenas uma pequena parte de um fenômeno socialmente significativo que ocorre no país, relacionado ao uso seguro de medicamentos. Para além da subnotificação dos casos de reações adversas a medicamentos e sua marcada heterogeneidade entre as regiões brasileiras, cabe salientar o seguinte fator importante: identificou-se baixa frequência de realização da investigação de causalidade dos pares medicamento-reação adversa entre os registros analisados, um dos objetivos primordiais de um sistema de farmacovigilância de um país.

\section{Colaboradores}

D. M. Mota contribuiu na concepção, análise e interpretação dos resultados, além da elaboração da redação científica do manuscrito. A. Vigo colaborou na concepção, análise e interpretação dos resultados, além da revisão final do conteúdo intelectual. R. S. Kuchenbecker contribuiu na concepção, análise e interpretação dos resultados, além da revisão crítica do conteúdo intelectual

\section{Informações adicionais}

ORCID: Daniel Marques Mota (0000-0003-41560138); Álvaro Vigo (0000-0002-5797-9470); Ricardo de Souza Kuchenbecker (0000-0002-47073683).

\section{Referências}

1. Al Hamid A, Ghaleb M, Aljadhey H, Aslanpour Z. A systematic review of hospitalization resulting from medicine-related problems in adult patients. Br J Clin Pharmacol 2014; 78:202-17.

2. Shepherd G, Mohorn P, Yacoub K, May DW. Adverse drug reaction deaths reported in United States Vital Statistics, 1999-2006. Ann Pharmacother 2012; 46:169-75.

3. Mota DM, Melo JRR, Freitas DRC, Machado M. Perfil da mortalidade por intoxicação com medicamentos no Brasil, 1996-2005: retrato de uma década. Ciênc Saúde Colet 2012; 17:61-70.

4. Lazarou J, Pomeranz BH, Corey PN. Incidence of adverse drug reactions in hospitalized patients: a meta-analysis of prospective studies. JAMA 1998; 279:1200-5.

5. Batel Marques F, Penedones A, Mendes D, Alves C. A systematic review of observational studies evaluating costs of adverse drug reactions. Clinicoecon Outcomes Res 2016; 8:41326.

6. Martins ACM. Eventos adversos a medicamentos: bancos de dados administrativos de pacientes hospitalizados e registro de óbitos como fonte de informação [Tese de Doutorado]. Rio de Janeiro: Escola Nacional de Saúde Pública Sergio Arouca, Fundação Oswaldo Cruz; 2015.

7. Edwards IR, Biriell C. Harmonisation in pharmacovigilance. Drug Saf 1994; 10:93-102. 
8. Bandekar MS, Anwikar SR, Kshirsagar NA. Quality check of spontaneous adverse drug reaction reporting forms of different countries. Pharmacoepidemiol Drug Saf 2010; 19:1181-5.

9. Singh A, Bhatt P. Comparative evaluation of adverse drug reaction reporting forms for introduction of a spontaneous generic ADR form. J Pharmacol Pharmacother 2012; 3:228.

10. Hazell L, Shakir SW. Under-reporting of adverse drug reactions: a systematic review. Drug Saf 2006; 29:385-96.

11. Silva JC, Soares M, Martins S. Reações adversas a medicamentos: análise da base de dados do Sistema Nacional de Farmacovigilância (SVIG). Relatório final. Lisboa: Autoridade $\mathrm{Na}$ cional do Medicamento e Produtos de Saúde, Ministério da Saúde; 2012.

12. Petramale CA. Projeto Hospitais Sentinela: uma estratégia de vigilância para a pós-comercialização de produtos de saúde. http://repo sitorio.enap.gov.br/bitstream/handle/1/483/ Projeto Hospitais Sentinelas.pdf? sequence $=1$ (acessado em 28/Mar/2016).

13. World Health Organization. Glossary of terms used in Pharmacovigilance. http://www.whoumc.org/graphics/27400.pdf (acessado em 15/ Nov/2014).

14. Agência Nacional de Vigilância Sanitária. Diretrizes para o gerenciamento do risco em farmacovigilância. Brasília: Agência Nacional de Vigilância Sanitária; 2008.

15. WHO Collaboration Centre for International Drug Monitoring. The WHO Adverse Reaction Terminology - WHO-ART. 2005. https://www.who-umc.org/vigibase/services/ learn-more-about-who-art/ (acessado em 07/ Jun/2016).

16. WHO Collaboration Centre for Drug Statistics Methodology. ATC/DDD Index 2016. http:// www.whocc.no/atc_ddd_index/ (acessado em 07/Jun/2016).

17. Mota DM, Vigo A, Kuchenbecker RS. Análise da preferência digital de idade no sistema de farmacovigilância do Brasil, 2008-2013. Ciênc Saúde Colet 2018; 23:3935-46.

18. Aagaard L, Strandell J, Melskens L, Petersen PSG, Holme Hansen E. Global patterns of adverse drug reactions over a decade. Drug Saf 2012; 35:1171-82.

19. Maigetter K, Pollock AM, Kadam A, Ward K, Weiss MG. Pharmacovigilance in India, Uganda and South Africa with reference to WHO's minimum requirements. Int $\mathrm{J}$ Health Policy Manag 2015; 4:295-305.

20. Agência Nacional de Vigilância Sanitária. Blog da Rede Sentinela, 2013. http://redesentinelaanvisa.blogspot.com.br/p/sobre-rede-sentine la.html (acessado em 06/Jun/2016).

21. Coêlho HL, Arrais PSD, Gomes AP. Sistema de farmacovigilância do Ceará: um ano de experiência. Cad Saúde Pública 1999; 15:631-40.

22. Vallano A, Cereza G, Pedròs C, Agustí A, Danés I, Aguilera C, et al. Obstacles and solutions for spontaneous reporting of adverse drug reactions in the hospital. Br J Clin Pharmacol 2005; 60:653-8.
23. Kongkaew C, Noyce PR, Ashcroft DM. Hospital admissions associated with adverse drug reactions: a systematic review of prospective observational studies. Ann Pharmacother 2008; 42:1017-25.

24. Agência Nacional de Vigilância Sanitária. RDC no 4, de 10 de fevereiro de 2009. Dispõe sobre as normas de farmacovigilância para os detentores de registro de medicamentos de uso humano. http://bvsms.saude.gov.br/bvs/saudele gis/anvisa/2009/res0004_10_02_2009.html (acessado em 21/Nov/2014).

25. Wysowski DK, Swartz L. Adverse drug event surveillance and drug withdrawals in the United States, 1969-2002: the importance of reporting suspected reactions. Arch Intern Med 2005; 165:1363-9.

26. Madurga M, Lázaro F, Martín-Serrano G, Quiroga M. El Sistema Español de Farmacovigilancia de Medicamentos de Uso Humano: su historia en cifras. In: Manso G, Hidalgo A, Carvajal A, Abajo FJ, editors. Los primeiros 25 años del Sistema Español de Farmacovigilancia de Medicamentos de Uso Humano. Oviedo: Universidad de Oviedo; 2010. p. 31-46.

27. Ferner RE. Determining causality case by case. In: World Health Organization, editor. Causality assessment in an evolving pharmacovigilance landscape. Conference report. Uppsala: Uppsala Monitoring Centre Research Conference; 2012. p. 7.

28. Ampadu HH, Hoekman J, Bruin ML, Pal SN, Olsson S, Sartori D, et al. Adverse drug reaction reporting in Africa and a comparison of individual case safety report characteristics between Africa and the rest of the world: analyses of spontaneous reports in VigiBase. Drug Saf 2016; 39:335-45.

29. Council for International Organizations of Medical Sciences. Current challenges in pharmacovigilance: pragmatic approaches. Geneva: Council for International Organizations of Medical Sciences; 2001. (Report of CIOMS Working Group V).

30. Rademaker M. Do women have more adverse drug reactions? Am J Clin Dermatol 2001; 2:349-51.

31. McDowell SE, Coleman JJ, Ferner RE. Systematic review and meta-analysis of ethnic differences in risks of adverse reactions to drugs used in cardiovascular medicine. BMJ 2006; 332:1177-81.

32. Goldman SA. Limitations and strengths of spontaneous reports data. Clin Ther 1998; 20 Suppl 3:C40-4.

33. Meyboom RH, Egberts AC, Gribnau FW, Hekster YA. Pharmacovigilance in perspective. Drug Saf 1999; 21:429-47.

34. Bennett CL. Thrombotic thrombocytopenic purpura associated with ticlopidine: a review of 60 cases. Ann Intern Med 1998; 128:541. 


\section{Abstract}

The study aimed to analyze suspected adverse drug reactions reported to Brazil's pharmacovigilance system (Notivisa-medicamento) from 2008 to 2013. This was a descriptive study whose analytical units in the database were reports and drug-adverse reactions pairs. A total of 26,554 reports were identified, for a reporting rate of 22.8/ million inhabitants/year. Reports in female patients prevailed (60.5\%), as did white color/race (58. $1 \%)$. Age ranged from 0 to 112 years (median $=$ 46 years). Nearly one-third (32.5\%) of suspected adverse drug reactions occurred in vulnerable populations (elderly and children). A total of 54,288 drug-adverse reactions pairs were analyzed, with a prevalence of severe adverse drug reactions (59.2\%), featuring those that resulted in clinically important effects (83.1\%). The most frequent drugs in severe adverse drug reactions belonged to groups $L$, antineoplastic and immunomodulating agents (32.1\%), and J, general anti-infectious agents for systemic use (27\%), while the most affected systemorgan-class was conditions of the skin and related disorders (23.7\%). Notivisa-medicamento is an important resource for producing warning signs and hypotheses on the safety of drugs marketed in Brazil. However, the reporting rate per million inhabitants/year was far lower than that described in middle and high-income countries.

Drug-Related Side Effects and Adverse Reactions; Pharmacoepidemiology; Pharmacovigilance; Adverse Drug Reactions Reporting Systems

\section{Resumen}

El objetivo de este estudio es analizar las sospechas de reacciones adversas a medicamentos, notificadas en el sistema de farmacovigilancia de Brasil (Notivisa-medicamento), entre 2008 y 2013. Se trata de un estudio descriptivo cuyas unidades de análisis en la base de datos fueron las notificaciones y los pares medicamento-reacción adversa. Se identificaron un total de 26.554 notificaciones, generando una tasa de notificación de 22,8/ millones de habitantes/año. Las notificaciones referentes al sexo femenino $(60,5 \%)$ prevalecieron, así como, la raza/color blanco (58,1\%). La edad varió de 0 a 112 años (mediana = 46 años). Casi 1/3 (32,5\%) de las sospechas de reacciones adversas a medicamentos ocurrieron en poblaciones vulnerables (ancianos y niños). Se evaluaron 54.288 registros de pares de medicamento-reacción adversa, donde prevalecieron las reacciones adversas graves (59,2\%), destacando las que resultaron en un efecto clínicamente importante (83,1\%). Los medicamentos más frecuentes en las reacciones adversas a medicamentos graves pertenecieron a los grupos $L$ - agentes neoplásicos e inmunomoduladores $(32,1 \%)$ y $J$ - antiinfecciosos generales para uso sistémico (27\%), mientras que el sistema-órganoclase más afectado fue afecciones de la piel y disturbios afines (23,7\%). El Notivisa-medicamento es una herramienta relevante para la producción de señales de alerta e hipótesis sobre la seguridad de medicamentos comercializados en Brasil. No obstante, la tasa de notificación por millón de habitantes/año se mostró muy inferior a la que está descrita en países de media y alta renta.

Efectos Colaterales y Reacciones Adversas Relacionados con Medicamentos;

Farmacoepidemiología; Farmacovigilancia; Sistemas de Registro de Reacción Adversa a Medicamentos
Recebido em 28/Jul/2018

Versão final reapresentada em 01/Fev/2019 Aprovado em 15/Mar/2019 